\title{
ERRATUM
}

Rosy Raman • Harsh Raman - Katie Johnstone •

Chris Lisle · Alison Smith · Peter Martin · Helen Allen

\section{Genetic and in silico comparative mapping of the polyphenol oxidase gene in bread wheat (Triticum aestivum L.)}

Received: 7 July 2005 / Accepted: 7 July 2005 / Published online: 15 September 2005

(C) Springer-Verlag 2005

Unfortunately the name of Dr. Peter Martin was misspelled in the original article.

The original article can be found at http://dx.doi.org/10.1007/s10142-005-0144-3

R. Raman $\cdot$ H. Raman $(\triangle) \cdot$ K. Johnstone $\cdot$ C. Lisle $\cdot$ A. Smith

P. Martin · H. Allen

NSW Department of Plant Industries

and NSW Agricultural Genomics Centre,

Wagga Wagga Agricultural Institute,

Wagga Wagga, NSW, 2650, Australia

e-mail: harsh.raman@agric.nsw.gov.au

Tel.: +61-02-69381925

Fax: +61-02-69381822

H. Raman

NSW Department of Primary Industries,

Wagga Wagga Agricultural Institute,

Private Mail Bag,

Wagga Wagga, NSW, 2650, Australia 